\title{
Determinación de concentraciones de cadmio en plantaciones de Theobroma cacao L. en Costa Rica ${ }^{1}$ Determination of cadmium concentrations in cocoa plantations (Theobroma cacao L.) in Costa Rica
}

Parménides Furcal-Beriguete ${ }^{2}$, José Luis Torres-Morales ${ }^{3}$

Furcal-Beriguete, P; Torres-Morales, J. Determinación de concentraciones de cadmio en plantaciones de Theobroma cacao L. en Costa Rica. Tecnología en Marcha. Vol. 33-1. Enero-Marzo. Pág 122-137.

doi) https://doi.org/10.18845/tm.v33i1.5027

1 Este trabajo forma parte del proyecto de investigación 2151-028 financiado por la Vicerrectoría de Investigación y Extensión (VIE) del Tecnológico de Costa Rica y por la Fundación para el Fomento y Promoción de la Investigación y Transferencia de Tecnología Agropecuaria de Costa Rica (FITTACORI).

2 Ingeniero Agrónomo, M.Sc. Escuela de Agronomía, Centro de Investigaciones y Desarrollo en Agricultura Sostenible del Trópico Húmedo (CIDASTH), Campus Local San Carlos, Instituto Tecnológico de Costa Rica. Correo electrónico: pafurcal@tec.ac.cr. (iD https://orcid.org/0000-0001-8695-4523

3 Ingeniero Agrónomo, Supervisor de Investigaciones, Dole, Costa Rica Correo electrónico: jose.l.torres@dole.com. 


\title{
Palabras clave
}

Metales pesados; cadmio en hoja de cacao; cadmio en raíz de cacao; cadmio en cacao.

\section{Resumen}

El cultivo de cacao se mantiene en auge en Costa Rica; sin embargo, las autoridades europeas han establecido regulaciones a la importación de los derivados del cacao; las cuales iniciaron en enero de 2019 con la fijación de niveles de concentración máximos permitidos de cadmio. El objetivo de este trabajo fue determinar concentraciones de cadmio en suelo, raíz, hoja y en grano seco no fermentado de cacao en dos regiones productoras de este cultivo en el país. El presente estudio exploratorio se realizó en 2017 y 2018, determinándose la presencia de cadmio en las partes raíz y hoja del árbol de cacao, y en el grano. La información generada permitió determinar que parte de la producción de cacao se encuentra localizada en un entorno donde los órganos raíz y hoja pueden bioacumular este metal y transferirlo a la mazorca donde se detectó concentraciones de cadmio en granos, en un rango de 0 a $8,70 \mathrm{mg} / \mathrm{kg}$. El porcentaje de muestras positivas en granos fue mayor en la Región Sur con 89,47\%, mientras que en la Norte fue $33,33 \%$. Sin embargo, en los análisis de suelos hechos en las muestras de los mismos sitios donde se muestrearon los órganos del árbol, solo se encontró en la Región Sur con un porcentaje muy bajo de muestras positivas, tres muestras de un total de 19, quizás debido a que el límite mínimo de detección para suelo propuesto por el laboratorio fue de $1 \mathrm{mg} / \mathrm{kg}$.

\section{Keywords}

Heavy metals; cadmium in cocoa leaf; cadmium in cocoa root; cadmium in cocoa.

\begin{abstract}
Cocoa cultivation is booming in Costa Rica; however, European authorities have established regulations for the importation of cocoa derivatives; which started in January 2019 with the fixation of maximum allowed concentration levels of cadmium. The objective of this study was to determine concentrations of cadmium in soil, root, leaf and non-fermented dry cocoa beans in two regions producing this crop in the country. The present exploratory study carried out in 2017 and 2018, determining the presence of cadmium in the root and leaf parts of the cacao tree, and in the grain. The information generated allowed finding that part of the cocoa production is located in an environment where the root and leaf organs can bioaccumulate this metal and transfer it to the cob where the concentrations of cadmium were detected in beans in a range of 0 to $8,70 \mathrm{mg} / \mathrm{kg}$. The percentage of positive samples in beans was higher in the South Region with $89.47 \%$, while in the Northern it was $33.33 \%$. However, in the analyzes of soils made in the samples from the same sites where the tree organs were sampled, only a very low percentage of positive samples were found in the Southern Region, three samples out of a total of 19, perhaps due the minimum detection limit for soil proposed by the laboratory was $1 \mathrm{mg} / \mathrm{kg}$.
\end{abstract}

\section{Introducción}

\section{Antecedentes}

La producción de cacao en Costa Rica se concentra en pequeños productores, localizados en las Regiones Caribe, especialmente en Talamanca y Limón, Huetar Norte en Upala y Guatuso, y la Brunca o Sur; las fincas de mayor tamaño están conformadas por plantaciones cuyas áreas oscilan 
entre 60 y 110 ha, en el país se registran entre 4491 y 4750 ha sembradas [13]. La producción de cacao en el país es mayormente para exportación, dirigida principalmente al mercado europeo, en presentaciones de productos de chocolates, grano seco, pasta o licor, manteca y polvo de cacao, donde el menor porcentaje es de cacao en grano [12], aunque también se exporta a Estados Unidos y otros países, sin embargo, el país importa granos de cacao.

La Comisión Técnica Científica de Contaminantes de la Cadena Alimentaria (CONTAM) de la EFSA (Autoridad Europea de Seguridad Alimentaria), determinó que "La exposición alimentaria media al cadmio ( $\mathrm{Cd}$ ) en los países europeos se acerca o supera ligeramente la ingesta semanal tolerable de $2,5 \mathrm{\mu g} / \mathrm{kg}$ de peso corporal" y, en algunos subgrupos de la población, llega casi a duplicarse; esta Comisión indica que es necesario reducir la exposición al cadmio en la población [16]. A raíz de lo anterior, inicia el tema del cadmio en el cacao y sus derivados. El 12 de mayo del 2014, fue aprobado el Reglamento 488/2014, que modifica el Reglamento 1881/2006 de la Comisión, donde se añade más productos a la lista de productos controlados, entre ellos los derivados del cacao.

\section{Justificación}

Las autoridades europeas establecieron regulaciones a la importación de los derivados del cacao, las cuales se programaron para ser aplicadas a partir de enero de 2019 con la fijación de niveles máximos (NM) de concentración permitidos de cadmio $(\mathrm{Cd})$, a través de la Dirección General de Salud y Protección al Consumidor de la Unión Europea. A pesar que las regulaciones no son aplicadas a las exportaciones del grano, sí las afectan, puesto que los importadores utilizan estos límites para aplicarlos o castigar los precios que se definen en las bolsas de productos de Nueva York y de Londres [28].

Existe una fuerte correlación entre el contenido de metales pesados en el suelo y la concentración en los vegetales, por lo que hay un riesgo asociado por el consumo de vegetales y la biodisponibilidad de los metales pesados, al ser estos cultivados en áreas contaminadas [1]. También, la disponibilidad de metales pesados, incluyendo el $\mathrm{Cd}$, en granos de cacao es impulsada por actividades antrópicas [29, 32]; además de estas influencias que aumentan las concentraciones en el ambiente, los metales pesados se encuentran de forma natural en la corteza terrestre $[5,26]$. Es decir que el factor principal que interviene en el contenido de $\mathrm{Cd}$ es el material parental; el promedio fluctúa entre 0,2-1,1 mg/kg [23]. En Ecuador se reporta, a través de estudios, un nivel crítico en el grano de cacao seco y fermentado de $1 \mathrm{mg} / \mathrm{kg}$ [26].

En el 2016 se finalizó el primer informe generado en el país, por el Programa de Investigación y Transferencia de Tecnología Agropecuaria (PITTA) en cacao y el Instituto Nacional de Innovación y Transferencia en Tecnología Agropecuaria (INTA), realizado en granos fermentados y secos de cacao [19], sin embargo, se debe estudiar más para disponer de otras informaciones que permitan ampliar el horizonte en este tema a nivel de finca de los productores. A partir de la información anterior, surge el proyecto "Determinación de la concentración de Cd en el suelo y órganos del árbol de cacao en las regiones productoras de cacao en Costa Rica", con el objetivo de determinar el contenido de cadmio en suelo, en las partes vegetativas raíz y hoja, y en los granos secos no fermentados del árbol de cacao (Theobroma cacao L.).

\section{Materiales y Métodos}

\section{Ubicación del proyecto}

Este artículo cubre dos de las tres regiones productoras de cacao del país, representadas por la Región Norte, donde las fincas visitadas se ubican entre 41 y 477 msnm y la Región Sur con fincas muestreadas en alturas desde 15 a 601 msnm. Este estudio exploratorio se realizó entre 
2017 y 2018, iniciándose con la selección de las fincas a través de un listado de productores de cacao suministrado por PITTA cacao y las recomendaciones de los funcionarios de las Agencias del Ministerio de Agricultura y Ganadería (MAG).

Se diseñó un cuestionario para recolección de información de cada productor, relacionada con la aplicación de insumos en el suelo y los árboles de cacao, además de la suministrada por los funcionarios del MAG.

Área experimental y unidad de medición

El área experimental estuvo constituida por el total de fincas muestreadas, 21 y 19 fincas en las Regiones Norte y Sur, respectivamente, para un total de 40 muestras de suelos, de grano y de cada una de las partes raíz y hoja en árboles no menores a cinco años. Para efecto de este estudio, cada finca constituyó una repetición, se seleccionaron al menos cinco árboles o unidades de observación en los cuales se registraron las variables analizadas para cada finca.

\section{Muestreo de suelos, raíces, hojas y mazorcas de cacao}

Las submuestras se tomaron en dos puntos en el suelo, en al menos cinco árboles, a partir de los $20 \mathrm{~cm}$ desde tronco del árbol hasta una distancia donde se proyecta el centro de la copa del árbol, a una profundidad de $20 \mathrm{~cm}$. La recolección se hizo por medio de un barreno tipo holandés y de un palín y depositado en un recipiente de hule donde se homogenizaron y cuartearon para conformar una sola muestra compuesta por finca o por lote de la finca.

Las muestras fueron procesadas en el Laboratorio de Análisis Agronómicos (LAA) del ITCR, estas se dejaron secar a temperatura ambiente, se molieron y tamizaron a $2 \mathrm{~mm}$ de diámetro. Los análisis efectuados en el LAA del ITCR fueron materia orgánica y granulométrico. El análisis químico se hizo en el Centro de Investigaciones Agronómicas (CIA) de la Universidad de Costa Rica, en el cual fue determinada acidez, $\mathrm{pH}$ y elementos esenciales; el cadmio se realizó con un plasma de acoplamiento inductivo (ICP) a un espectrofotómetro óptico, con muestra previamente digerida con ácido nítrico y ácido fluorhídrico.

Las raíces se recolectaron desde los $20 \mathrm{~cm}$ del tronco alrededor del mismo hasta donde se proyectaba la copa del árbol, a una profundidad de $20 \mathrm{~cm}$ entre los puntos de muestreos del suelo. Estas fueron introducidas en bolsas plásticas y transportadas dentro de una hielera con suficiente hielo para su conservación.

Las hojas se seleccionaron cinco por cada árbol, para formar una muestra de al menos 25 hojas por lote muestreado. Se tomaron en la sección media de la copa del árbol con hojas expuesta al sol y a la sombra, en la sección de las ramas con hojas sanas y maduras fisiológicamente. Estas muestras fueron transportadas hasta el laboratorio bajo las mismas condiciones que las raíces, luego de ser procesadas e identificadas fueron enviadas al laboratorio de suelos y

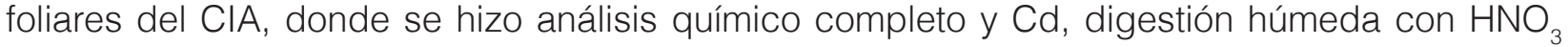
y determinación a través de plasma de acoplamiento inductivo (ICP) a un espectrofotómetro óptico.

Se colectó una mazorca madura de cacao en cada uno de los árboles donde se tomaron las muestras de suelos, raíces y hojas y se trasladaron para su procesamiento en el laboratorio. De las mazorcas muestreadas se procedió a eliminar la placenta y el mucílago que, luego se lavaron las semillas con agua, se mezclaron y por último se introdujeron en el horno a una temperatura de $60^{\circ} \mathrm{C}$ y aire forzado durante 96 horas, se procedió al molido de las semillas con las cáscaras mediante la utilización de un mortero y pistilo para luego tamizarlas. 


\section{Resultados y discusión}

De acuerdo a los resultados, las dos regiones tienen en mayor o menor grado la presencia de cadmio $(\mathrm{Cd})$ en los órganos del árbol de cacao. La región con mayor presencia es la Sur con $84,21 \%$ de las 19 muestras en la raíz y la hoja, y 89,47\% en el grano, distribuido en toda la región, excepto en Pérez Zeledón que no apareció, además fue la región con presencia de este metal en tres de las 19 muestras de suelos, no obstante en la Región Norte no se detectó $\mathrm{Cd}$ en los suelos con un límite mínimo de detección de $1 \mathrm{mg} / \mathrm{kg}$ establecido por el laboratorio (cuadro 1).

Cuadro 1. Resumen de estadística descriptiva de los resultados de las muestras analizadas para explorar la presencia de cadmio en tres regiones de Costa Rica.

\begin{tabular}{|c|c|c|c|c|c|c|c|}
\hline & \multicolumn{9}{|c|}{ Estadística } \\
\hline \multirow{4}{*}{ Región Norte } & Suelo/órgano & $\mathrm{n}$ & Promedio & Mínimo & Máximo & Des. Estándar & $\%$ positivo \\
\cline { 2 - 8 } & Suelo & 21 & $\mathrm{ND}$ & $\mathrm{ND}$ & $\mathrm{ND}$ & $\mathrm{ND}$ & ND \\
\cline { 2 - 8 } & Raíz & 21 & 0,34 & 0,00 & 1,50 & 0,55 & 28,57 \\
\cline { 2 - 8 } & Hoja & 21 & 0,65 & 0,00 & 3,10 & 0,90 & 38,01 \\
\cline { 2 - 8 } & Grano & 21 & 0,44 & 0,00 & 1,80 & 0,64 & 33,33 \\
\hline \multirow{3}{*}{ Región Sur } & Suelo & 19 & 0,38 & 0,00 & 4,00 & 0,99 & 15,79 \\
\cline { 2 - 8 } & Raíz & 19 & 2,44 & 0,00 & 13,40 & 2,80 & 84,21 \\
\cline { 2 - 8 } & Hoja & 19 & 2,71 & 0,00 & 11,10 & 2,44 & 84,21 \\
\cline { 2 - 8 } & Grano & 19 & 2,25 & 0,00 & 8,70 & 2,06 & 89,47 \\
\hline
\end{tabular}

ND: no detectado con un límite mínimo de detección de $1 \mathrm{mg} / \mathrm{kg}$.

\section{Resultados y discusión de la Región Norte}

$\mathrm{pH}$ de los suelos de la Región Norte

El 33\% de los valores de $\mathrm{pH}$ en los suelos corresponden a valores entre 4,8 y 5,5 de pH, el restante 67\% (14 muestras) se encuentra con pH entre 5,6 y 6,3 (cuadro 2). De acuerdo a [3] se clasifican como suelos muy ácidos y ácidos.

El pH ácido es considerado el factor más importante en aumentar la solubilidad de los metales pesados y su disponibilidad en la concentración en la solución del suelo [10, 18, 33, 35]. Del mismo modo, [31] y [20] mencionan que valores por debajo de 5,5 es donde se encuentra la mayor disponibilidad del $\mathrm{Cd}$, sin embargo, esta disponibilidad depende de características propias del metal como movilidad, forma química, concentraciones del elemento en el suelo, tipo de suelo, presencia de otros metales y fuente productora de Cd [31].

Por lo anterior, los valores de pH de esta zona pueden ser propicios para que el Cd se encuentre soluble. En suelos alcalinos, el Cd es removido del suelo y adsorbido por los coloides lo que hace que su biodisponibilidad disminuya considerablemente [14]. Suelos que contienen $\mathrm{CaCO}_{3}$ puede existir un intercambio de la molécula de $\mathrm{Ca}$ con la de $\mathrm{Cd}$ pasando a formar $\mathrm{CdCO}_{3}$, siempre que existan concentraciones altas de Cd en el suelo [10]. 
Cuadro 2. Resultados de análisis químicos para cadmio y fertilidad de suelos de la Región Norte de Costa Rica.

\begin{tabular}{|c|c|c|c|c|c|c|c|c|c|c|c|}
\hline \multirow[b]{2}{*}{ Código } & \multirow[b]{2}{*}{$\mathrm{pH}$} & \multicolumn{4}{|c|}{$\mathrm{cmol}(+) / \mathrm{L}$} & \multicolumn{5}{|c|}{$\mathrm{mg} / \mathrm{L}$} & \multirow{2}{*}{$\frac{\mathrm{mg} / \mathrm{kg}}{\mathrm{Cd}}$} \\
\hline & & $\begin{array}{c}\text { Acidez } \\
\text { extractable }\end{array}$ & $\mathrm{Ca}$ & $\mathrm{Mg}$ & K & $\mathrm{Cu}$ & $\mathrm{Mn}$ & $\mathrm{Fe}$ & $\mathrm{Zn}$ & $P$ & \\
\hline \multicolumn{12}{|c|}{ Cantón Guatuso } \\
\hline S-2-15-03-01-1-L1 & 5,6 & 0,24 & 5,52 & 1,5 & 0,12 & 9 & 18 & 107 & 3,7 & 2 & ND \\
\hline S-2-15-03-01-1-L2 & 5,6 & 0,30 & 4,26 & 1,0 & 0,07 & 10 & 19 & 117 & 3,9 & 1 & ND \\
\hline S-2-15-01-01-1 & 5,5 & 0,62 & 4,53 & 1,7 & 0,09 & 12 & 127 & 168 & 3,8 & 2 & ND \\
\hline S-2-15-04-01-1 & 5,5 & 0,19 & 5,50 & 1,3 & 0,24 & 17 & 125 & 130 & 4,7 & 2 & ND \\
\hline S-2-15-02-01-1 & 6,3 & 0,11 & 8,60 & 3,0 & 0,11 & 13 & 168 & 96 & 4,7 & 3 & ND \\
\hline S-2-15-02-01-2 & 5,4 & 0,17 & 6,28 & 1,8 & 0,20 & 18 & 197 & 142 & 5,5 & 2 & ND \\
\hline S-2-15-04-01-2 & 5,8 & 0,13 & 8,79 & 3,0 & 0,16 & 13 & 127 & 142 & 6,9 & 2 & ND \\
\hline S-2-15-04-01-3 & 6,1 & 0,10 & 13,6 & 4,9 & 0,47 & 14 & 43 & 157 & 3,8 & 7 & ND \\
\hline \multicolumn{12}{|c|}{ Cantón Upala } \\
\hline S-2-13-03-01-1 & 6,3 & 0,12 & 11,4 & 4,7 & 0,46 & 12 & 31 & 107 & 3,9 & 5 & ND \\
\hline S-2-13-03-01-2 & 6,2 & 0,11 & 15,1 & 5,9 & 0,34 & 10 & 41 & 51 & 8,1 & 3 & ND \\
\hline S-2-13-03-01-3 & 6,2 & 0,10 & 14,3 & 4,8 & 0,51 & 18 & 31 & 99 & 5,2 & 5 & ND \\
\hline S-2-13-03-01-4 & 5,8 & 0,10 & 21,2 & 7,0 & 0,27 & 14 & 35 & 56 & 3,6 & 4 & ND \\
\hline S-2-13-07-01-1 & 6,1 & 0,12 & 19,3 & 5,4 & 0,70 & 8 & 36 & 46 & 4,0 & 4 & ND \\
\hline S-2-13-07-01-2 & 5,7 & 0,15 & 19,3 & 5,1 & 0,64 & 8 & 31 & 93 & 6,7 & 6 & ND \\
\hline S-2-13-07-01-3 & 5,6 & 0,13 & 25,9 & 9,8 & 0,26 & 8 & 39 & 40 & 8,6 & 3 & ND \\
\hline S-2-13-04-01-1 & 5,3 & 0,26 & 5,04 & 1,0 & 0,31 & 9 & 19 & 99 & 3,4 & 14 & ND \\
\hline S-2-13-04-01-2 & 5,3 & 0,28 & 7,83 & 2,7 & 0,22 & 10 & 88 & 108 & 2,4 & 1 & ND \\
\hline S-2-13-04-01-3 & 4,8 & 0,43 & 6,50 & 1,6 & 0,50 & 19 & 19 & 130 & 4,08 & 6 & ND \\
\hline \multicolumn{12}{|c|}{ Cantones San Carlos- Los Chiles } \\
\hline S-2-14-02-01-1 & 5,2 & 0,22 & 6,84 & 2,6 & 0,17 & 10 & 202 & 154 & 3,5 & 1 & ND \\
\hline S-2-10-13-01-1 & 5,2 & 0,22 & 5,93 & 2,6 & 0,12 & 14 & 118 & 203 & 3,6 & 2 & ND \\
\hline S-2-10-11-01-1 & 5,0 & 1,06 & 3,24 & 1,2 & 0,17 & 8 & 38 & 313 & 2,7 & 2 & ND \\
\hline
\end{tabular}

ND: no detectado con un límite mínimo de detección de $1 \mathrm{mg} / \mathrm{kg}$

El $52 \%$ de los productores visitados utiliza alguna fuente de fertilizante, pero su uso no es mayor de dos veces por año, como para poder alterar alguna característica química del suelo.

Materia orgánica (MO) de los suelos de la Región Norte

Los contenidos de MO varían en un rango de 1,69 a 8,86 (cuadro 3), valores mayores o iguales a $5 \%$ de $\mathrm{MO}$ en suelos que no sean de origen volcánico son considerados buenos [9], por otro lado, para el cultivo de cacao valores iguales o superiores a $3 \%$ en $\mathrm{MO}$ son aceptables para el 
establecimiento del cultivo [25]. La Región Norte mostró un 33 \% (siete muestras) de las fincas con valores que van desde un $1 \%$ a $3 \%$ de $\mathrm{MO}$, el restante $67 \%$ (14 muestras) mostraron valores entre $3 \%$ y $8,86 \%$ (cuadro 3). Se encontró que un solo productor utiliza abono orgánico como parte de la fertilización del cultivo, la finca de este productor obtuvo 4,28\% de MO.

Los ácidos húmicos y fúlvicos que aumentan en el proceso de humificación de la MO tienen un papel importante en la capacidad de formar enlaces con los grupos fenólicos y carboxílicos con mayor capacidad y fuerza de retención [11, 20,34], de esta manera afecta la disponibilidad del Cd para las plantas. La formación de los complejos organometálicos entre el Cd y la MO es más estable conforme aumenta el pH, por lo que a mayor $\mathrm{pH}$ la movilidad y solubilidad de este metal será menor [20].

\section{Análisis granulométrico de los suelos de la Región Norte}

Los resultados de análisis granulométrico y de textura en este estudio indica la dominancia de suelos arcillosos (cuadro 3).

Las arcillas tienen un papel muy importante en la adsorción de los metales, las cargas superficiales negativas, son responsables de la capacidad de intercambio catiónico del suelo y a su vez son encargadas de disminuir o aumentar la liberación de los metales [2, 21, 33]; además del contenido, el tipo de arcilla tiene un rol fundamental, ya que estas se comportan de formas diferentes [31].

Cuadro 3. Resultados de análisis de materia orgánica y granulométrico en suelos de Región Norte de Costa Rica.

\begin{tabular}{|c|c|c|c|c|c|}
\hline \multirow[b]{2}{*}{ Código } & \multicolumn{4}{|c|}{$\%$} & \multirow[b]{2}{*}{ Nombre Textural } \\
\hline & $\mathrm{MO}$ & Arena & Arcilla & Limo & \\
\hline \multicolumn{6}{|c|}{ Cantón Guatuso } \\
\hline S-2-15-03-01-1-L1 & 7,40 & 42 & 42 & 16 & Arcilloso \\
\hline S-2-15-03-01-1-L2 & 8,86 & 57 & 27 & 16 & Franco A. Arenoso \\
\hline S-2-15-01-01-1 & 2,86 & 19 & 62 & 19 & Arcilloso \\
\hline S-2-15-04-01-1 & 3,47 & 19 & 67 & 14 & Arcilloso \\
\hline S-2-15-02-01-1 & 4,72 & 9 & 69 & 21 & Arcilloso \\
\hline S-2-15-02-01-2 & 3,62 & 9 & 70 & 21 & Arcilloso \\
\hline S-2-15-04-01-2 & 4,28 & 17 & 60 & 24 & Arcilloso \\
\hline S-2-15-04-01-3 & 2,12 & 27 & 32 & 41 & Franco Arcilloso \\
\hline \multicolumn{6}{|c|}{ Cantón Upala } \\
\hline S-2-13-03-01-1 & 2,74 & 16 & 52 & 32 & Arcilloso \\
\hline S-2-13-03-01-2 & 2,26 & 24 & 44 & 32 & Arcilloso \\
\hline S-2-13-03-01-3 & 3,27 & 49 & 32 & 20 & Franco A. Arenoso \\
\hline S-2-13-03-01-4 & 3,73 & 29 & 42 & 29 & Arcilloso \\
\hline S-2-13-07-01-1 & 1,69 & 29 & 42 & 29 & Arcilloso \\
\hline S-2-13-07-01-2 & 3,34 & 31 & 39 & 30 & Franco Arcilloso \\
\hline S-2-13-07-01-3 & 2,46 & 24 & 52 & 25 & Arcilloso \\
\hline S-2-13-04-01-1 & 3,40 & 36 & 47 & 17 & Arcilloso \\
\hline S-2-13-04-01-2 & 3,57 & 24 & 57 & 19 & Arcilloso \\
\hline S-2-13-04-01-3 & 3,64 & 26 & 57 & 17 & Arcilloso \\
\hline \multicolumn{6}{|c|}{ Cantones San Carlos- Los chiles } \\
\hline S-2-14-02-01-1 & 1,87 & 24 & 62 & 14 & Arcilloso \\
\hline S-2-10-13-01-1 & 3,95 & 11 & 77 & 12 & Arcilloso \\
\hline S-2-10-11-01-1 & 4,77 & 16 & 69 & 14 & Arcilloso \\
\hline
\end{tabular}




\section{Contenido de zinc en los suelos de la Región Norte}

El contenido de zinc en los suelos analizados, se muestra en un rango desde 2,4 mg/L a 8,6 mg/L (cuadro 2). Además del pH, cantidad de materia orgánica, y el porcentaje y tipo de arcilla, el nivel de zinc (Zn) en el suelo es considerado de alta importancia respecto al Cd. En la Región Norte el 90\% de las 19 muestras presentaron valores superiores a $3 \mathrm{mg} / \mathrm{L}$ del elemento, valor considerado como nivel crítico en cuanto a fertilidad de los suelos.

El comportamiento entre estos dos elementos se produce principalmente por su similitud química, cuando se da una liberación en la fase solida del suelo ambos metales compiten por las sedes de sorción, así la presencia de Zn en el medio afecta la adsorción del Cd [31]. La absorción del $\mathrm{Cd}$ a nivel radicular está en competencia específica con otros nutrientes como el manganeso, cobre, zinc, hierro y calcio [22]. Por el contrario, [2] indica, que existe un efecto sinérgico entre el Zn y el Cd, de igual modo [17] demostró en tres suelos diferentes que la adición conjunta de $\mathrm{Zn}$ y $\mathrm{Cd}$ muestran un efecto sinérgico entre ambos elementos, donde elevadas concentraciones de Zn favorecen la movilidad del $\mathrm{Cd}$.

\section{Contenido de cadmio en suelos de la Región Norte}

El contenido de Cd en el suelo no fue detectado (cuadro 2) con un límite mínimo de detección (LMD) en suelo de $1 \mathrm{mg} / \mathrm{kg}$ establecido por el laboratorio; la concentración de cadmio en suelo se encuentra de 0,1 a $1 \mathrm{mg} / \mathrm{kg}[2,8]$.

De acuerdo a la literatura citada, la disponibilidad de Cd para la planta es menor en suelos con alto contenido de materia orgánica, de arcillas y de zinc. Bajo estas condiciones algunas fincas de la Región Norte tienen bajo porcentaje de MO, y pH ácido, lo que favorece la disponibilidad del Cd, sin embargo, en estos suelos no se detectó Cd con un LMD de $1 \mathrm{mg} / / \mathrm{kg}$.

\section{Resultados análisis de cadmio en raíz, hoja y grano de cacao en la Región Norte}

Según los resultados obtenidos, a nivel de raíz se encontró la presencia de Cd en seis muestras, lo que representa un 28,57 \% de las 21 analizadas (cuadro 4), con valores que van desde 1,0 a $1,5 \mathrm{mg} / \mathrm{kg}$; en raíz el contenido depende de la cantidad del elemento que se encuentre disponible en el suelo [38]. Las concentraciones de metales en las raíces podrían proporcionar mejor indicador de biodisponibilidad respecto al suelo [24], debido, en parte, al aumento de exudados alrededor de la raíz y a la actividad microbiana inducida por estos que aumentan la solubilidad y biodisponibilidad del metal.

Cuando el $\mathrm{Cd}$ se encuentra disponible en la solución del suelo puede ser absorbido por la planta y acumulado en los diferentes órganos [5]. La entrada del Cd a la raíz se puede dar por cargas negativas, en parte, generadas por el grupo carboxilo proveniente del ácido péptico y las cargas positivas de los metales en el suelo [27]; no obstante, [22] menciona que la absorción del Cd a nivel radicular está en competencia específica con otros nutrientes como el manganeso, cobre, zinc, hierro y calcio. El Cd penetra la raíz por medio del tejido cortical y es translocado a los demás tejidos de la planta [37].

La presencia de Cd en raíz en algunas muestras, a pesar de no detectarse en suelo por debajo del límite de $1 \mathrm{mg} / \mathrm{kg}$, puede ser debido a que inferior a este valor en el suelo puede existir, además que las plantas son capaces de acidificar el suelo alrededor de las raíces modificando la actividad de la enzima $\mathrm{H}^{+}$ATPasa por medio de la producción de exudados carboxilados haciendo biodisponibles los metales presentes en el suelo, que pueden ser absorbidos por la planta y almacenados en los diferentes órganos; de igual forma el Cd puede penetrar a la raíz utilizando transportadores de otros metales tales como el calcio, hierro y zinc [30]. 
Cuadro 4. Resultados de análisis de cadmio en raíz, hoja y grano no fermentado y seco de Theobroma cacao L. de la Región Norte de Costa Rica.

\begin{tabular}{|c|c|c|c|}
\hline & \multicolumn{3}{|c|}{$\mathrm{Cd}(\mathrm{mg} / \mathrm{kg})$} \\
\hline & Raíz & Hoja & Grano seco \\
\hline Código & \multicolumn{3}{|c|}{ Cantón Guatuso } \\
\hline 2-15-03-01-1-L1 & ND & ND & ND \\
\hline 2-15-03-01-1-L2 & ND & ND & ND \\
\hline 2-15-01-01-1 & ND & 1,5 & 1,0 \\
\hline $2-15-04-01-1$ & ND & 1,3 & 1,1 \\
\hline $2-15-02-01-1$ & ND & ND & ND \\
\hline $2-15-02-01-2$ & ND & ND & ND \\
\hline $2-15-04-01-2$ & ND & ND & ND \\
\hline $2-15-04-01-3$ & 1,2 & 1,4 & 1,6 \\
\hline \multicolumn{4}{|c|}{ Cantón Upala } \\
\hline 2-13-03-01-1 & 1,3 & 1,8 & 1,1 \\
\hline 2-13-03-01-2 & ND & ND & ND \\
\hline $2-13-03-01-3$ & ND & ND & ND \\
\hline 2-13-03-01-4 & 1,2 & 1,8 & 1,5 \\
\hline $2-13-07-01-1$ & 1,0 & ND & ND \\
\hline $2-13-07-01-2$ & 1,0 & 1,4 & ND \\
\hline $2-13-07-01-3$ & 1,5 & 3,1 & 1,8 \\
\hline $2-13-04-01-1$ & ND & 1,4 & 1,1 \\
\hline $2-13-04-01-2$ & ND & ND & ND \\
\hline $2-13-04-01-3$ & ND & ND & ND \\
\hline \multicolumn{4}{|c|}{ Cantón San Carlos } \\
\hline $2-14-02-01-1$ & ND & ND & ND \\
\hline $2-10-13-01-1$ & ND & ND & ND \\
\hline $2-10-11-01-1$ & ND & ND & ND \\
\hline
\end{tabular}

ND: No detectado con un límite mínimo de detección de $1 \mathrm{mg} / \mathrm{kg}$.

En tejido foliar el contenido de Cd fue encontrado en ocho de las 21 muestras en la Región Norte (38\%), los valores encontrados van desde 1,3 a 3,1 mg/kg (cuadro 4). El límite permisible de cadmio en tejido foliar es de $0,5 \mathrm{mg} / \mathrm{kg}[2,24]$. El Cd es liberado al ambiente mediante erupciones volcánicas, transporte de partículas del suelo por el viento, quemas forestales, por otro lado, actividades humanas como minería, actividades industriales entre ellas fundición de metales, uso de fertilizantes fosfatados, entre otras, también favorecen la liberación de este metal [30]; asimismo, [24] mencionan que este metal puede ser absorbido vía foliar por la planta, por lo que no necesariamente todo el Cd encontrado dentro de la planta tiene que ser originario del suelo. Sin embargo, las fincas donde se hicieron los análisis no se encuentran en zonas con riesgos de haber sido afectadas por contaminación ambiental de origen industrial o de transporte, además en la encuesta aplicada se detectó que muy pocos propietarios aplican fertilizantes orgánicos o inorgánicos con frecuencia mayor a una vez al año, tampoco el cultivo se encuentra con técnica de irrigación, por lo que las únicas posibilidades serían por origen de los suelos y en algunas localidades por posibles emanaciones volcánicas. 
Uno de los métodos que puede desarrollar la planta en defensa contra el Cd hacen que esta logre inmovilizar este metal en los tricomas de las hojas, [36], quizás esta es la razón que en la Región Norte la hoja, por lo general, es el órgano de mayor acumulación de Cd.

La detección de $\mathrm{Cd}$ en el grano juega un rol importante por ser la parte de consumo de la planta. Un 33\% de las muestras (siete) posee contenido de Cd en el grano de cacao no fermentado y seco, estas se encuentran con valores que van de 1 a $1,8 \mathrm{mg} / \mathrm{kg}$ (cuadro 4). El nivel máximo permisible de Cd en grano es de 0,5 mg/kg [15, 37]. El Cd una vez absorbido por la raíz se une a la pared celular de las células epidérmicas para luego ser transportado por intercambio iónico al resto de órganos de la planta [30], esta información concuerda con [7] que indica que la planta de cacao absorbe los metales que existen en el suelo y los tiende a acumular en los granos; mientras que, en algunas etapas de la planta, tales como crecimiento, floración y formación de fruto se prioriza el trasporte de elementos a esas partes para cumplir y proporcionar a la planta la capacidad de poder optimizar su desarrollo [4].

De las seis muestras con Cd en la raíz, cinco de ellas presentaron en la hoja. Asimismo, de las ocho muestras con Cd en las hojas, siete de ellas aparecieron con el elemento en el grano, esto nos permite inferir que el proceso de translocación en la planta de este elemento se produjo desde la raíz hacia los demás órganos y por ende se da su acumulación en el grano (cuadro 4), este resultado se confirma con las correlaciones existentes (cuadro 5). En ese sentido, además se refuerza la tesis que, aunque no se detectó Cd en las muestras de suelos con un LMD de 1 $\mathrm{mg} / \mathrm{kg}$, existe la posibilidad que los suelos contengan este elemento por debajo de este límite y las raíces sean capaces de absorberlo y acumularlo a través del tiempo, raíces de árboles que fueron muestreados con mínimo de cinco años, edad suficiente para tener metales acumulados.

\section{Correlaciones del contendido de cadmio entre las variables evaluadas en la Región Norte}

Se encontró correlación positiva muy significativa entre el Cd encontrado en el tejido foliar, en el grano y el Cd en la raíz. Igualmente existe correlación positiva, pero menos fuerte que las demás, entre el contenido de $\mathrm{Cd}$ en la raíz y el grano de cacao $(0,6256)$ con p-valor $=0,0032$ (cuadro 5), lo que quiere decir que si hay un aumento en los contenidos de cadmio en raíz sucedería lo mismo en la concentración del elemento en la hoja, lo mismo que entre la hoja y el grano. [6] y [7] mencionan que la planta transporta el cadmio absorbido y lo almacena en los granos.

\section{Resultados y discusión de la Región Sur}

En la zona sur se muestrearon un total de 19 fincas distribuidas en cuatro cantones, los resultados obtenidos en los análisis de suelos evidencian niveles de $\mathrm{pH}$ muy similares entre fincas, predominancia de textura arcillosa y un rango amplio en porcentaje de materia orgánica (cuadros 6 y 7). Se detectó cadmio en el suelo en un total de tres fincas con valores de 1.3, 1.9 y $4 \mathrm{mg} / \mathrm{kg}$ (cuadro 6), los cuales son superiores a la concentración de 0,1 a $1 \mathrm{mg} / \mathrm{kg}$ de este elemento en el suelo propuesto por [2] y [8].

Cuadro 5. Análisis de correlación entre contenido de cadmio en las diferentes partes analizadas de la planta en la Región Norte de Costa Rica.

\begin{tabular}{|c|c|c|c|}
\hline & Cd (Raíz) & Cd (Hoja) & Cd (Grano) \\
\hline Cd (Raíz) & 1 & 0,0004 & 0,0032 \\
\hline Cd (Hoja) & 0,7194 & 1 & $5,10 \mathrm{E}-08$ \\
\hline Cd (Grano) & 0,6256 & 0,9029 & 1 \\
\hline
\end{tabular}


Cuadro 6. Resultados de análisis químicos de suelos de la Región Sur de Costa Rica.

\begin{tabular}{|c|c|c|c|c|c|c|c|c|c|c|c|}
\hline \multirow[b]{2}{*}{ Código } & \multirow[b]{2}{*}{$\mathrm{pH}$} & \multicolumn{4}{|c|}{$\mathrm{cmol}(+) / \mathrm{L}$} & \multicolumn{5}{|c|}{$\mathrm{mg} / \mathrm{L}$} & \multirow{2}{*}{$\begin{array}{c}\mathrm{mg} / \mathrm{kg} \\
\mathrm{Cd}\end{array}$} \\
\hline & & $\begin{array}{c}\text { Acidez } \\
\text { extractable }\end{array}$ & $\mathrm{Ca}$ & $\mathrm{Mg}$ & K & $\mathrm{Cu}$ & $\mathrm{Mn}$ & $\mathrm{Fe}$ & $\mathrm{Zn}$ & $P$ & \\
\hline \multicolumn{12}{|c|}{ Cantón Osa } \\
\hline $6-05-05-01-1$ & 5,2 & 1,43 & 20 & 3,8 & 0,2 & 4 & 90 & 80 & 3 & 1 & ND \\
\hline 6-05-05-01-2-L1 & 5,2 & 0,6 & 33,9 & 3 & 0,1 & 4 & 39 & 77 & 6,2 & 2 & ND \\
\hline 6-05-05-01-2-L2 & 6,6 & 0,12 & 49 & 3,1 & 0,2 & 5 & 19 & 33 & 10 & 5 & 1,3 \\
\hline 6-05-05-01-2-L3 & 5,7 & 0,25 & 36,6 & 3,9 & 0,2 & 8 & 90 & 48 & 6,3 & 5 & ND \\
\hline 6-05-01-01-1 & 5,1 & 0,81 & 19,8 & 3,53 & 0,49 & 10 & 33 & 168 & 2,0 & 6 & ND \\
\hline $6-05-01-01-2$ & 5,6 & 0,40 & 28,24 & 4,71 & 0,24 & 18 & 62 & 118 & 2,0 & 5 & ND \\
\hline $6-05-01-01-3$ & 5,2 & 0,92 & 22,3 & 5,13 & 0,47 & 10 & 51 & 150 & 4,0 & 11 & ND \\
\hline $6-05-05-01-2$ & 5,5 & 0,59 & 33,36 & 4,27 & 0,66 & 181 & 20 & 112 & 3,7 & 33 & ND \\
\hline $6-05-05-01$ & 6,8 & 0,12 & 43,9 & 3,17 & 0,59 & 12 & 13 & 46 & 1,9 & 7 & ND \\
\hline \multicolumn{12}{|c|}{ Cantón Golfito } \\
\hline 6-07-01-01-1 & 5,7 & 0,26 & 35,5 & 5,8 & 0,3 & 110 & 46 & 90 & 6,1 & 7 & ND \\
\hline $6-07-03-01-2$ & 5,2 & 2,35 & 27,1 & 6,8 & 0,3 & 4 & 54 & 39 & 3 & 1 & ND \\
\hline $6-07-03-01-1$ & 5,4 & 1,09 & 31,8 & 5,3 & 0,2 & 4 & 51 & 49 & 2,5 & 1 & ND \\
\hline \multicolumn{12}{|c|}{ Cantón Corredores } \\
\hline $6-10-01-01-1$ & 5,5 & 0,27 & 22,8 & 6,3 & 0,4 & 5 & 63 & 45 & 3,1 & 2 & 1,9 \\
\hline $6-10-01-01-2$ & 5,6 & 0,25 & 19,7 & 1,9 & 0,7 & 6 & 45 & 71 & 1,8 & 2 & 4.0 \\
\hline $6-10-01-12$ & 5,4 & 0,65 & 26,44 & 4,23 & 0,61 & 5 & 54 & 50 & 2,3 & 2 & ND \\
\hline $6-10-01-12$ & 5,5 & 1,21 & 14,95 & 3,14 & 0,55 & 5 & 26 & 192 & 2 & 16 & ND \\
\hline $6-10-01-01-2$ & 5,3 & 2,66 & 11,62 & 2,82 & 0,38 & 5 & 35 & 182 & 1,5 & 1 & ND \\
\hline \multicolumn{12}{|c|}{ Cantón Pérez Zeledón } \\
\hline $1-19-02-01-1$ & 4,7 & 1,65 & 0,90 & 0,26 & 0,11 & 11 & 11 & 298 & 2 & 11 & ND \\
\hline $1-19-05-01-1$ & 4,8 & 1,63 & 3,05 & 0,89 & 0,41 & 7 & 21 & 133 & 13 & 4 & ND \\
\hline
\end{tabular}

ND: No detectado con un límite mínimo de detección de $1 \mathrm{mg} / \mathrm{kg}$.

El aumento en la solubilidad del $\mathrm{Cd}$ depende del $\mathrm{pH}[20,31]$, también esta disponibilidad depende de características propias del metal como movilidad, forma química, concentraciones del elemento en el suelo, tipo de suelo, presencia de otros metales y materiales fuentes del cadmio [31].

En nuestro caso la relación $\mathrm{Cd}-\mathrm{pH}$ no se cumplió, ya que las tres fincas a las cuales se les detectó este elemento en el suelo poseen $\mathrm{pH}$ igual o superior a 5,5, aunque siguen siendo suelos ácidos. El 29\% de las fincas muestreadas utiliza algún tipo de abono químico granular como Rocas fosfóricas, 10-30-10, Kmag y urea, pero no más de dos veces al año. 
Materia orgánica de las muestras de suelos en Región Sur

El valor mínimo considerado adecuado para el cultivo de cacao es 3\% [25], 18 muestras se encuentran por encima de este valor representando el 94,73\% de los suelos muestreados. Resultados altos de MO podrían favorecer la fijación de los metales en el suelo y por lo tanto afecta la absorción del cadmio por parte de la planta [11, 20,34]. sin embargo, no se informa por encima de cual valor se puede fijar el Cd

\section{Análisis granulométrico y textura de los suelos en Región Sur}

Las condiciones obtenidas en este estudio favorecen la fijación de cadmio con las arcillas (cuadro 7), lo que trae como consecuencia una posible disminución en su disponibilidad, mediante la formación de enlaces, formados principalmente, por las diferencias entre las cargas eléctricas de las arcillas y del metal [2, 21, 33].

Cuadro 7. Resultados de análisis de materia orgánica y granulométrico en suelos de la Región Sur de Costa Rica.

\begin{tabular}{|c|c|c|c|c|c|}
\hline \multirow[b]{2}{*}{ Código } & \multicolumn{4}{|c|}{$\%$} & \multirow[b]{2}{*}{ Nombre Textural } \\
\hline & $\mathrm{MO}$ & Arena & Arcilla & Limo & \\
\hline \multicolumn{6}{|c|}{ Cantón Osa } \\
\hline $6-05-05-01-1$ & 2,64 & 21 & 59 & 19 & Arcilloso \\
\hline 6-05-05-01-2-L1 & 6,10 & 16 & 62 & 22 & Arcilloso \\
\hline 6-05-05-01-2-L2 & 8,20 & 24 & 64 & 12 & Arcilloso \\
\hline 6-05-05-01-2-L3 & 4,30 & 27 & 62 & 12 & Arcilloso \\
\hline $6-05-01-01-1$ & 4,93 & 34 & 37 & 29 & Franco Arcilloso \\
\hline $6-05-01-01-2$ & 4,48 & 27 & 37 & 36 & Franco Arcilloso \\
\hline $6-05-01-01-3$ & 4,91 & 30 & 42 & 29 & Arcilloso \\
\hline $6-05-05-01-2$ & 3,48 & 34 & 32 & 31 & Franco Arcilloso \\
\hline $6-05-05-01$ & 3,30 & 49 & 30 & 29 & Franco Arcilloso \\
\hline \multicolumn{6}{|c|}{ Cantón Golfito } \\
\hline $6-07-01-01-1$ & 3,40 & 9 & 57 & 34 & Arcilloso \\
\hline $6-07-03-01-2$ & 4,10 & 34 & 42 & 24 & Arcilloso \\
\hline $6-07-03-01-1$ & 3,50 & 31 & 44 & 25 & Arcilloso \\
\hline \multicolumn{6}{|c|}{ Cantón Corredores } \\
\hline $6-10-01-01-1$ & 7,20 & 26 & 57 & 17 & Arcilloso \\
\hline $6-10-01-01-2$ & 6,00 & 31 & 54 & 15 & Arcilloso \\
\hline 6-10-01-12 & 3,02 & 34 & 45 & 21 & Arcilloso \\
\hline 6-10-01-12 & 5,70 & 49 & 35 & 16 & Franco A. Arenoso \\
\hline $6-10-01-01-2$ & 6,29 & 37 & 45 & 29 & Arcilloso \\
\hline \multicolumn{6}{|c|}{ Cantón Pérez Zeledón } \\
\hline $1-19-02-01-1$ & 7,00 & 7 & 80 & 14 & Arcilloso \\
\hline $1-19-05-01-1$ & 10,09 & 47 & 34 & 19 & Franco A. Arenoso \\
\hline
\end{tabular}


Resultados de análisis Cd en raíz, hojas y granos de cacao de la Región Sur

En los resultados obtenidos en los análisis de Cd en raíz y hojas se detectó el metal en 16 $(84,21 \%)$ de las 19 muestras, en raíz el valor mínimo fue de 1,10 mg/kg y un máximo 13,4 mg/ $\mathrm{kg}$, el valor promedio y de la mediana fue de 3,01 y de 2,46 mg/kg, respectivamente (cuadro 8).

La formación de exudados alrededor de las raíces favorece algunas condiciones de suelos que ayudan a la disponibilidad del Cd y la absorción por la raíz [30], y por ende su translocación a las partes aéreas de la planta.

Cuadro 8. Resultados análisis de cadmio en raíz, hoja y grano no fermentado de Theobroma cacao L. Región Sur de Costa Rica.

\begin{tabular}{|c|c|c|c|}
\hline & \multicolumn{3}{|c|}{$\mathrm{Cd} \mathrm{mg} / \mathrm{kg}$ ) } \\
\hline & Raíz & Hoja & Grano seco \\
\hline Código & \multicolumn{3}{|c|}{ Cantón de Osa } \\
\hline $6-05-05-01-1$ & 3,30 & 3,10 & 2,00 \\
\hline 6-05-05-01-2-L1 & 1,90 & 3,00 & 2,90 \\
\hline 6-05-05-01-2-L2 & 2,40 & 3,80 & 5,00 \\
\hline 6-05-05-01-2-L3 & 2,60 & 4,40 & 4,10 \\
\hline $6-05-01-01-1$ & ND & ND & 1,10 \\
\hline $6-05-01-01-2$ & 1,10 & 1,40 & 1,40 \\
\hline $6-05-01-01-3$ & 1,30 & 1,90 & 2,00 \\
\hline $6-05-05-01-2$ & 2,86 & 4,58 & 2,44 \\
\hline $6-05-05-01$ & 2,52 & 4,49 & 2,45 \\
\hline \multicolumn{4}{|c|}{ Cantón de Golfito } \\
\hline 6-07-01-01-1 & 3.00 & 2,80 & 1,60 \\
\hline 6-07-03-01-2 & 2,60 & 1,60 & 1,60 \\
\hline $6-07-03-01-1$ & 1,60 & 1,80 & 1,20 \\
\hline \multicolumn{4}{|c|}{ Cantón de Corredores } \\
\hline 6-10-01-01-1 & 3,80 & 3,00 & 4,50 \\
\hline 6-10-01-01-2 & 13,40 & 11,10 & 8,70 \\
\hline $6-10-01-12$ & 1,31 & 1,23 & 0,60 \\
\hline $6-10-01-12$ & 1,18 & 1,23 & 0,70 \\
\hline 6-10-01-01-2 & 1,46 & 2,02 & 0,56 \\
\hline \multicolumn{4}{|c|}{ Cantón El General } \\
\hline $1-19-02-01-1$ & ND & ND & ND \\
\hline 1-19-05-01-1 & ND & ND & ND \\
\hline
\end{tabular}

ND: No detectado con un límite de detección de $1 \mathrm{mg} / \mathrm{kg}$.

El valor mínimo en hoja fue de 1,23 mg/kg y un máximo de11,10 mg/kg (cuadro 8); el promedio fue de 3,44 y la mediana de 3,00 mg/kg. Los valores de Cd en las hojas están por encima 0,5 $\mathrm{mg} / \mathrm{kg}$, límite máximo permisible en tejido foliar [2] [24]; el 36,84\% de los productores utilizan 
algún tipo de insumo de origen químico vía foliar, sin embargo, la frecuencia de utilización es baja (una o dos aplicaciones al año), además fincas que no usan insumos presentan contenido de Cd. Las mismas muestras con presencia de Cd en la raíz son las mismas con Cd en la hoja, por lo tanto, los procesos de translocación del elemento en la planta movilizan el Cd desde la raíz hasta la hoja del árbol, una vez absorbido se moviliza por diferentes rutas hasta llegar a los distintos órganos [36]; bajo este criterio, si no existe posibilidad de contaminación del aire y no ha sido aplicado el elemento por insumos vía foliar, entonces existe una alta probabilidad que el origen del Cd en la raíz, hoja y grano, sea el suelo.

La concentración de $\mathrm{Cd}$ en el grano se encontró dentro de un rango muy amplio que va de 0,56 a $8,7 \mathrm{mg} / \mathrm{kg}$ (cuadro 8), con promedio de 2,31 y mediana de 2,00 mg/kg, y porcentaje de presencia alto, igual que los demás órganos (cuadro 1); la planta de cacao tiende a acumular metales pesados en los granos [7].

\section{Resultados de correlación en la Región Sur}

El cadmio que se encuentra disponible en el suelo puede ser absorbido por la planta y acumulado en los diferentes órganos $[5,37]$. Es posible que debido a lo anterior se registren las correlaciones altas presentes en el cuadro 9.

Cuadro 9. Análisis de correlación entre contenido de cadmio en los suelos y las diferentes partes analizadas de la planta de la Región Sur de Costa Rica.

\begin{tabular}{|c|c|c|c|c|}
\hline & Cd (Suelos) & Cd (Raíz) & Cd (Hoja) & Cd (Grano) \\
\hline Cd (Suelos) & 1 & 0,00000061 & 0,000092 & 0,0000013 \\
\hline Cd (Raíz) & 0,881 & 1 & 0,0000000052 & 0,00000019 \\
\hline Cd (Hoja) & 0,780 & 0,930 & 1 & 0,0000001 \\
\hline Cd(Grano) & 0,870 & $\mathbf{0 , 8 6 0}$ & $\mathbf{0 , 9 0 0}$ & 1 \\
\hline
\end{tabular}

\section{Conclusiones}

Del total de muestras de suelos analizadas (40), solo tres resultaron positivas con cadmio, correspondientes a la Región Sur, en las restantes no se detectó el elemento con un límite de detección de $1 \mathrm{mg} / \mathrm{kg}$.

En raíz, la presencia de cadmio fue $28,57 \%$ y $84,21 \%$ para un número de muestras analizadas de 21 y 19 en las Regiones Norte y Sur, respectivamente. Mientras que, para el mismo número de muestras y regiones, en el órgano hoja la presencia de cadmio fue 38,01\% y 84,21\%. De igual manera, en el grano de cacao no fermentado y seco, las dos regiones resultaron positivas a la presencia de cadmio con 33,33\% y 89,47\%, respectivamente.

Se encontró que, a pesar de las pocas muestras de suelos que resultaron positivas al cadmio, la presencia de este elemento en los órganos del árbol de cacao fue una realidad, mayormente en la Región Sur, lo que sugiere que hubo una bioacumulación de este metal. Por lo tanto, se debe profundizar con estudios en los suelos con un límite de detección de cadmio más bajo que el propuesto en este trabajo y en análisis de granos fermentados y secos por lotes específicos en los centros de acopio. 


\section{Referencias}

[1] Agbenin, J., Danko, M., y Welp, G. 2009. Soil and vegetable composition relationships of eight potentially toxic metals in urban garden fields from northern Nigeria. J. Sci Food Agr. 89:49-54: doi 10.1002/jsfa.3409.

[2] Alloway, B. 2002. Heavy metal in soil. Third Edition. Editorial Board. Canada. 614p.

[3] Andrade, M. y Martínez, M. 2014. Fertilidad del suelo y parámetros que la definen. Consultado el 31 de julio de 2018. Disponible en file://C:/Users/Jos\%C3\%A9\%20Luis\%20Torres/Desktop/Dialnet-FertilidadDelSueloYP arametrosQueLaDefinen-267902.pdfArboleda

[4] Arce, M. 2017. Efecto de las relaciones fuente: sumideros decrecientes sobre la expresión sexual y la carga de frutos de papaya (Carica papaya L.) hibrido Pococí. Tesis Lic. Agronomía. Universidad de Costa Rica. 98p

[5] Arévalo, E., Obando, M., Zúñiga, L., Arévalo, C., Baligar, B. y He, Z. 2016. Metales pesados en suelos de plantaciones de cacao (Theobroma cacao. L) en tres regiones del Perú. Ecología aplicada. 15: 81-89

[6] Augstburger, F., Berger, J., Censkowsky, U., Heid, P. \& Milz, J. 2000. Agricultura Orgánica en el trópico y subtrópico. En: Guía de 18 cultivos. 1ra Edición. Alemania. 24p.

[7] Barrueta, S. 2013. Guía de métodos de detección y análisis de cadmio en cacao (Theobroma cacao L.). Consultado 09 mayo 2018. Disponible en https://issuu.com/riicchperu/docs/guia_de_metodos_de_ deteccion_y_anal

[8] Beltran, M. 2001. Fitoextracción en suelos contaminados con Cadmio y Zinc usando especies vegetales comestibles. Tesis M. Sc. Ingeniería Ambiental. México. Universidad Autónoma metropolitana. 176h.

[9] Bertsch, F. 1987. Manual para interpretar la fertilidad de los suelos en Costa Rica. San José. Costa Rica. 83p.

[10] Boulding, R. 1996. Enviromental assesment sourcebook. USA. 400p.

[11] Bravo, I., Arboleda, C., Martin, F. 2014. Efecto de la calidad de la materia organica asociada con el uso y manejo de suelos en la retención de cadmio en sistemas alcalinos de Colombia. Acta Agronomica. 63(2):1-14

[12] Canacao (Asociación Cámara Nacional de Cacao Fino de Costa Rica). 2016. Biblioteca. Cacao de Costa Rica. Consultado 18 may. 2016.

[13] Carmiol G., G. 2017. Análisis de la actividad cacaotera costarricense y perspectivas de su reactivación. SEPSA (Secretaria Ejecutiva de Planificación Sectorial Agropecuaria).2017-001. 82 p. Consultado el 11 mayo 2017. Disponible en http://www.sepsa.go.cr/DOCS/2017-001-Diagnostico_cacao.pdf

[14] Carrasco, A. 2005. Metales pesados en el suelo. Consultado el 30 de noviembre de 2017. Disponible en http://biblioteca-digital.sag.gob.cl/documentos/medio_ambiente/criterios_calidad_suelos_aguas_agricolas/ pdf_suelos/5_metales_pesados_suelo.pdf

[15] Contreras, F., Herrera, T., Izquierdo, A. 2011. Efecto de dos fuentes de carbonato de calcio $\left(\mathrm{CaCO}_{3}\right)$ sobre la disponibilidad de cadmio para plantas de cacao (Theobroma cacao L.) en suelos de Barlovento, estado de Miranda. Revista Venesuelos. 13:52-63.

[16] Diario Oficial de la Unión Europea. 2014. Reglamento (UE) No 488/2014 de la Comisión del 12 de mayo de 2014 que modifica el Reglamento (CE) № 1881/2006 por lo que respecta al contenido máximo de cadmio en los productos alimenticios. Diario Oficial de la Unión Europea.13 de mayo de 2014. L138/75-79.

[17] Estévez, J., Andrade, L., Marcet, P., Montero, J. 2000. Fijación y movilidad del cadmio y zinc en tres tipos de suelos ácidos de Galicia, España. Ciencia del suelo. 18(1):28-35.

[18] Galán, E., Romero, A. 2008. Contaminación de suelos por metales pesados. Consultado el 26 de noviembre de 2017. Disponible en http://www.ehu.eus/sem/macla_pdf/macla10/Macla10_48.pdf

[19] Gamboa, J. 2016. Determinación de concentraciones de cadmio en granos fermentados y secos de cacao (Theobroma cacao L.), en los cantones cacaoteros de Costa Rica. Código CC 01 NM 501-9-15. Instituto Nacional de Innovación y Transferencia de Tecnología Agropecuaria (INTA). Fundación para el fomento y promoción de la investigación Transferencia de tecnología agropecuaria de Costa Rica (FITTACORI). San José, Costa Rica. 39 p.

[20] García, C., Moreno, J., Hernández, L., Polo, A. 2002. Metales pesados y sus implicaciones en la calidad del suelo. Consultado el 29 de noviembre de 2017. Disponible en http://digital.csic.es/handle/10261/111812

[21] Hooda, P. 2010 Trace elements in soils. Londres. Inglaterra. 618p.

[22] Huamaní, H., Huauya, R., Mansilla, L., Florida, N., Neira, G. 2012. Presencia de metales pesados en cultivo de cacao (Theobroma cacao L.) orgánico. Acta Agronómica. 61(4):339-344.

[23] Kabata-Pendias, A. 2011. Trace elements in soils and plants. $4^{\text {th }}$ ed. CRC Press. Taylor and Francis Group. Boca Raton (FL). 505p.

[24] Kabata-Pendias, A. and Pendias, H. 2001. Trace elements in soil s and plants. $3^{\text {rd }}$ ed. CRC Press. Boca Raton, London New York, Washington, D.C.USA. 403p. 
[25] López, P; Ramírez, M., Mendoza, A. 2011. Paquete tecnológico cacao (Theobroma cacao.L) establecimiento y mantenimiento. Consultado el 03 de julio de 2018. Disponible en file:///C:/Users/Jos\%C3\%A9\%20Luis\%20 Torres/Desktop/cacao_establecimiento.pdf

[26] Mite, F., Carrillo, M., Durango, W. 2010. Avances del monitoreo de presencia de cadmio en almendras de cacao, suelos y aguas en Ecuador. XII Congreso Ecuatoriano de la Ciencia del Suelo. 17-19 de noviembre de 2010.21p. (en línea). Consultado 24 enero 2019. Disponible en http://www.secsuelo.org/wp-content/ uploads/2015/06/6.-Francisco-Mite.-Cadmio.-INIAP.pdf

[27] Navarro, J., Aguilar, L., López, J. 2007. Aspectos bioquímicos y genéticos de la tolerancia y acumulación de metales pesados en plantas. Ecosistemas. 16(2):10-25

[28] Organización Mundial del Comercio (OMC). G/SPS/GEN/1587 (17-5872). 2017. Reglamento (UE) № 488/2014 de la Comisión, del 12 de mayo de 2014, que modifica el Reglamento (CE) № 1881/2006 por lo que respecta al contenido máximo de cadmio en los productos alimenticios. Comunicación de Perú. 30 de mayo de 2017. P 1-2.

[29] Peris, M., Micó, C., Recatalá, L., Sánchez, R. y Sánchez, J. 2007. Heavy metal contents in horticultural crops of a representative area of the European Mediterranean region. Sci Total Environ.; 378(1-2):42-48.

[30] Pernía, B., De Sousa, A., Reyes, R., Castrillo, M. 2008. Biomarcadores de contaminación por cadmio en las plantas. Consultado el 06 de mayo de 2018. Disponible en http://www.redalyc.org:9081/home.oa?cid=958504

[31] Rábago, I., Aracil, J. 2011. Capacidad de amortiguación de la contaminación por plomo y por cadmio en suelos de la comunidad de Madrid. Tesis PhD Farmacia. Madrid, España. Universidad Complutense de Madrid $285 p$.

[32] Rato, J., Ramos-Miras, J., Lopez-Piñeiro, A., Loures, L.., Gil, C., Coelho, J., Loures, A. 2014. Concentrations of Available Heavy Metals in Mediterranean Agricultural Soils and their Relation with Some Soil Selected Properties: A Case Study in Typical Mediterranean Soils. Sustainability 2014, 6 (12) 9124-9138; https://doi. org/10.3390/su6129124.

[33] Rieuwerts, J., Thornton, I., Farago, M., Ashmore, M. 2015. Factors influencing metal bioavailability in soils: preliminary investigations for the development of a critical loads approach for metals. Chemical Speciation and Bioavailability. 10(2): 61-75

[34] Rodríguez, H. 2001. Estudio de la contaminación por metales pesados en la cuenca del rio Llobregat. Tesis $\mathrm{PhD}$ en Ingeniería Minera y recursos naturales. Catalunya. Universidad politécnica de Catalunya. $281 \mathrm{~h}$

[35] Sánchez, G. 2016. Eco toxicología del cadmio. Riesgo para la salud de la utilización de suelos ricos en cadmio. Tesis. Lic Farmacia. México. Universidad complutense.19h

[36] Serrano, M., Martínez, N., Romero, M., Del Rio, L., Sandallo, L. 2008. Toxicidad de cadmio en plantas. Consultado el 08 de mayo de 2018. Disponible en https://www.revistaecosistemas.net/index.php/ecosistemas/article/view/409

[37] Tantalean, E., Huauya, M. 2017. Distribución del contenido de cadmio en los diferentes órganos de cacao CCN-51 en suelo aluvial y residual en las localidades de Jacintillo y Ramal de Aspuzana. Revista de investigación agroproduccion sustentable 1(2): 69-78

[38] Wilquerson, J. 2017. Determinación de la absorción de cadmio y plomo en genotipos de cacao (Theobroma cacao L.) para el establecimiento de plantaciones comerciales. Tesis. Lic. Ing Agronómica. Perú. Universidad Nacional de San Martin-Tarapoto. 75h 